Pacific Journal of Mathematics

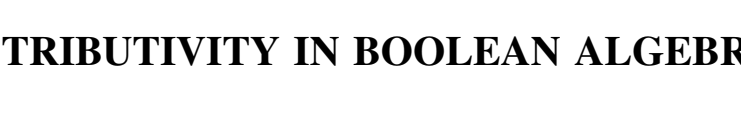




\title{
DISTRIBUTIVITY IN BOOLEAN ALGEBRAS
}

\author{
R. S. PIERCE
}

1. Introduction. Let $\alpha$ be an infinite cardinal number; suppose $B$ is an $\alpha$-complete Boolean algebra, that is, every subset of $\mathrm{B}$ which contains no more than $\alpha$ elements has a least upper bound in $B$.

Definition 1.1. $B$ is $\alpha$-distributive if the following identity ${ }^{1}$ holds in $B$ whenever $S$ and $T$ are index sets of cardinality $\leqq \alpha$ :

$$
\bigwedge_{\sigma \in S}\left(\bigvee_{\tau \in T} a_{\sigma \tau}\right)=\bigvee_{\varphi \in F}\left(\bigwedge_{\sigma \in S} a_{\sigma \varphi(\sigma)}\right), \text { where } F=T^{S}
$$

This paper studies $\alpha$-distributive Boolean algebras, their Boolean spaces and the continuous functions on these Boolean spaces. A survey of the main results can be obtained by reading Theorems $6.5,7.1,8.1$ and 8.2.

2. Notation. Throughout the paper, $\alpha$ denotes a fixed infinite cardinal number. The term $\alpha$-B.A. is used to abbreviate $\alpha$-complete Boolean algebra. Only $\alpha$-complete algebras are considered, although some of the definitions apply to arbitrary Boolean algebras. We speak of $\alpha$-subalgebras, $\alpha$-ideals, $\alpha$-homomorphisms, $\alpha$-fields, etc., meaning that the relevant operations enjoy closure up to the power $\alpha$. For example, an $\alpha$-field is a field of sets, closed under $\alpha$-unions, that is, unions of $\alpha$ or fewer element.

The lattice operations of join, meet and complement are designated by $\vee, \wedge$ and (') respectively. The symbols 0 and $u$ stand for the zero and unit elements of a Boolean algebra. Set operations are represented by rounded symbols: $\cup, \cap$ and $\leqq$ respectively denote union, intersection and inclusion. If $A$ and $B$ are sets, $B-A$ is the set of elements of $B$ which are not in $A$; the complement (in a fixed set) of $A$ is designated $A^{c}$. The empty set is denoted by 0 . The symbol $\overline{\bar{A}}$ stands for the cardinality of the set $A$. Finally, for typographical reasons, we use the symbols $2^{\alpha}$ and $\exp (\alpha)$ interchangeably.

Received February 17, 1956. The research in this paper was done, in part, while the author was a Jewett fellow of the Bell telephone laboratories.

1 The notion of $\alpha$-distributibity was introduced in [1]. It is assumed that the least upper bound an the right side of the equality (1) exists. However, by Corollary 3.4 below, it would suffice to make the equality in (1) contingent on the existence of this least upper bound. 


\section{Alternative characterizations of $\alpha$-distributivity.}

Definition 3.1. A subset $A$ of an $\alpha$-B.A. $B$ is called a covering of $B$ if l.u.b. $A=u$. The covering $A$ is called an $\alpha$-covering if $\overline{\bar{A}} \leqq \alpha$. A binary partition of $B$ is a pair consisting of an element of $B$ and its complement. If $A$ and $\tilde{A}$ are coverings of $B$, then $\tilde{A}$ is said to refine $A$ if every $\tilde{a} \in \tilde{A}$ is $\leqq$ some $a \in A$.

Proposition 3.2. Let $B$ be an $\alpha$-B.A. Then the following are equivalent properties of $B$ :

(i ) $B$ is $\alpha$-distributive ;

(ii) if $\left\{A_{\sigma} \mid \sigma \in S\right\}$ is a set of $\alpha$-converings of $B$ and $\overline{\bar{S}} \leqq \alpha$, then there exists a covering $A$ which refines every $A_{\sigma}$;

(iii) if $\left\{A_{\sigma} \mid \sigma \in S\right\}$ is a set of binary partitions of $B$ and $\overline{\bar{S}} \leqslant \alpha$, then there exists a covering $A$ which refines every $A_{\sigma}$.

Proof. ${ }^{2}$ (i) implies (ii). Indeed, if we index each $A_{\sigma}$ by a set $T$ of cardinality $\leqq \alpha$, say $A_{\sigma}=\left\{a_{\sigma \tau} \mid \tau \in T\right\}$ (allowing repetitions), then $\left\{\bigwedge_{\sigma \in S} a_{\sigma \varphi(\sigma)} \mid \varphi \in T^{S}\right\}$ is a covering which refines every $A_{\sigma}$.

(ii) implies (iii), obviously.

(iii) implies (i). Let $A_{\sigma \tau}=\left\{a_{\sigma \tau},\left(a_{\sigma \tau}\right)^{\prime}\right\}$ for all $\sigma \in S, \tau \in T$. Because the cardinality of $S \times T$ is $\leqq \alpha$, there exists a covering $A$ which refines every $A_{\sigma \tau}$. Suppose $0 \neq b \leqq \bigwedge_{\sigma \in S}\left(\bigvee_{\tau \in T} a_{\sigma \tau}\right)$. Since l.u.b. $A=u$, there exists $a \in A$ such that $a \wedge b \neq 0$. Then for each $\sigma \in S$, we can find $\tau \in T$ such that $a \wedge a_{\sigma \tau} \neq 0$. Denoting this $\tau$ by $\varphi(\sigma)$ defines $\varphi \in F=T^{S}$. But $A$ refines $A_{\sigma_{\varphi(\rho)}(\sigma)}$, so $a \leqq a_{\sigma \varphi(\sigma)}$ for all $\sigma$. Hence, $a \leqq \bigwedge_{\sigma \in S} a_{\sigma \varphi(\sigma)}$. It follows that $b \wedge\left(\wedge_{\sigma \in S} a_{\sigma \varphi(\sigma)}\right) \neq 0$. Since $b$ can be arbitrarily small, $\wedge_{\sigma \in S}\left(\bigvee_{\tau \in I} a_{\sigma_{\tau}}\right)$ is the least upper bound of the set $\left\{\wedge_{\sigma \in S} a_{\sigma \varphi(\sigma)} \mid \varphi \in F\right\}$, that is, (1) is satisfied.

COROLlary 3.3. An $\alpha$-B.A. is $\alpha$-distributive if and only if (1) is identically satisfied under the conditions $\overline{\bar{S}} \leqq \alpha, \overline{\bar{T}}=2$ and $\alpha_{\sigma 1}=\left(a_{\sigma_{2}}\right)^{\prime}$.

Proof. By the argument that leads from (i) to (ii) in 3.2, the hypotheses of 3.3 imply (iii) of 3.2 .

COROLlary 3.4. Let $B$ be an $\alpha$-B.A. which is not $\alpha$-distributive.

2 The referee has pointed out that there is overlap between the first part of this paper and the independent work of Smith and Tarski [5]. In particular, 3.3 and 3.4 appear in [5] as Theorems 2.5 and 2.2, while our Corollaries 6.5 and 6.6 are special cases of Theorem 3.6 in [5]. It is a pleasure to acknowledge the contribution of a conscientious referee to the improvement of this paper. 
Then there exists $b \neq 0$ in $B$ and $a$ set of pairs $\left\{\left[c_{\sigma 1}, c_{\sigma 2}\right] \leqq B \mid \sigma \in \overline{\bar{S}}, S \leqq \alpha\right\}$ such that $c_{\sigma 1} \wedge c_{\sigma^{2}}=0$ and $c_{\sigma_{1}} \vee c_{\sigma_{2}}=b$ for all $\sigma \in S$ and $\bigwedge_{\sigma \in S} c_{\sigma \varphi(\sigma)}=0$ for all $\varphi \in T^{s}(T=[1,2])$.

Proof. By 3.3, if $B$ is not $\alpha$-distributive, there exists a set of complementary pairs $\left\{\left[a_{\sigma_{1}}, a_{\sigma_{2}}\right] \mid \sigma \in S, \overline{\bar{S}} \leq \alpha\right\}$ such that the unit of $B$ is not the least upper bound of the set of elements $\wedge_{\sigma \in S} a_{\sigma \varphi(\sigma)}, \varphi \in T^{s}$. Thus, there exists $b \neq 0$ in $B$ such that $b \wedge\left(\bigwedge_{\sigma \in S} a_{\sigma \varphi(\sigma)}\right)=0$ for all $\varphi \in T^{s}$. Then $c_{\sigma 1}=b \wedge a_{\sigma 1}$ and $c_{\sigma_{3}}=b \wedge a_{\sigma_{2}}$ have the required properties.

4. Examples of $\alpha$-distributive Boolean algebras. Every $\alpha$-field of sets is $\alpha$-distributive. Moreover, from Definition 1.1.

(4.1) Every $\alpha$-subalgebra of an $\alpha$-distributive B.A. is $\alpha$-distributive ;

(4.2) Every $2^{\alpha}$-homomorph of an $\alpha$-distributive B.A. is $\alpha$-distributive.

Using (4.2), it is easy to construct $\alpha$-distributive algebras which are not $\alpha$-fields of sets (following Horn and Tarski [2, p. 492], or Sikorski $[4$, p. 253]): let $B$ be the B.A. of all subsets of a set $X$ with $\overline{\bar{X}}=$ $\exp (\exp (\alpha))$. Let $I$ be the $\alpha$-ideal of all subsets $M$ of $X$ such that $\overline{\bar{M}} \leqq \exp (\alpha)$. Then (see Tarski [8], or the remarks following 6.6 below), there exists no prime $\alpha$-ideal of $B$ which contains $I$, and consequently $B / I$ has no prime $\alpha$-ideals. Hence, $B / I$ is not an $\alpha$-field. On the other hand, by (4.2), $B / I$ is $\alpha$-distributive.

It is easy to see that (4.2) cannot be strengthened to assert that every $\alpha$-homomorphic image of an $\alpha$-field is $\alpha$-distributive. In fact, by the theorem of Loomis (see [3]), every $\boldsymbol{s}_{0}$-B.A. is the $\boldsymbol{H}_{0}$-homomorph of an -field. But not every $\kappa_{0}$-B.A. is $\kappa_{0}$-distributive: an atomless measure algebra in which all nonzero elements have positive measure is not $\widehat{N}_{0}$-distributive.

5. The representation of $\alpha$-distributive algebras. In this section, we show that every $\alpha$-distributive B.A. is the $\alpha$-homomorph of an $\alpha$ field. If $\alpha=2^{\beta}$, then by (4.2) any $\alpha$-homomorphic image of an $\alpha$-field is $\beta$-distributive. This shows (as Sikorski observed in [4]) that the class of $\alpha$-homomorphs of $\alpha$-fields is rather elite when $\alpha \geq \exp \left(\boldsymbol{\psi}_{0}\right)$.

For any Boolean algebra $B$, let $X(B)$ denote the Boolean space of $B$. Then the points of $X(B)$ are the prime ideals of $B$ and the topology is imposed by taking all the sets $X(a)=\{P \in X(B) \mid a \notin P\}$, with $a \in B$, as a neighborhood basis. As Stone [6] has shown, $X(B)$ is a totally disconnected, compact, Hausdorff space and the correspondence $a \rightarrow X(\alpha)$ is an isomorphism of $B$ onto the Boolean algebra of open-and-closed sets of $X(B)$.

Definition 5.1. A set $T \subseteq X(B)$ is called $\alpha$-nowhere dense if there 
is an $\alpha$-covering $A$ of $B$ such that $T=\left(\bigcup_{a \in A} X(a)\right)^{c}=\bigcap_{a \in A} X\left(a^{\prime}\right)$.

(5.2) A closed set $T \subseteq X(B)$ is topologically nowhere dense in $X(B)$ (that is, $T$ contains no open subset of $X(B)$ ) if and only if there is a covering $A$ of $B$ such that $T=\left(\bigcup_{a \in A} X(a)\right)^{c}$. In particular, the $\alpha$-nowhere dense sets are just the closed, nowhere dense sets which are $\alpha$-intersections of open ${ }^{3}$ sets.

LemMA 5.3. If $B$ is an $\alpha$-distributive B.A., and if $\left\{T_{\sigma} \mid \sigma \in S\right\}$ is a set of $\alpha$-nowhere dense sets in $X(B)$ with $\overline{\bar{S}} \leqq \alpha$, then $\bigcup_{\sigma \in S} T_{\sigma}$ is nowhere dense in $X(B)$.

Proof. By 5.1, $T_{\sigma}=\left(\cup_{a \in A_{\sigma}} X(\alpha)\right)^{c}$, where $A_{\sigma}$ is an $\alpha$-covering of $B$. By 3.2, there is a covering $A$ which refines every $A_{\sigma}$. Then $T=$ $\left(\bigcup_{a \in A} X(a)\right)^{c}$ is a nowhere dense set (by (5.2)) which contains every $T_{\sigma}$.

THEOREM 5.4. If $B$ is an $\alpha$-distributive B.A., then $B$ is the $\alpha$ homomorphic image of an $\alpha$-field of sets.

Proof. ${ }^{4}$ Let $F$ be the $\alpha$-field generated by the open-and-closed subsets of $X(B)$. Let $I$ be the $\alpha$-ideal of $F$ generated by the $\alpha$-nowhere dense subset of $X(B)$. Consider the collection $\tilde{F}$ of sets in $F$ which are congruent modulo $I$ to some $X(a)$ with $a \in B$. The $\alpha$-completeness of $B$ implies that $\tilde{F}$ is an $\alpha$-field; since $\tilde{F}$ contains every $X(a), \tilde{F}=F$. By 5.3, $X(a) \in I$ only if $a=0$. Hence, $F / I$ is isomorphic to $B$.

6. Quotients of $a$-distributive algebras. We wish now to characterize the ideals $I$ of an $\alpha$-distributive B.A. for which $B / I$ is $\alpha$-distributive.

Definition 6.1. Let $S$ be an index set with $\overline{\bar{S}} \leq \alpha$. For each $\sigma \in S$, suppose $A_{\sigma}=\left\{a_{\sigma \tau} \mid \tau \in T\right\}$ is a subset of the $\alpha$-B.A. B. Denote

$$
\prod_{\sigma \in S} A_{\sigma}=\left\{\bigwedge_{\sigma \in S} a_{\sigma \varphi(\sigma)}{ }^{\prime} \mid \varphi \in T^{S}\right\} \cup\{0\} .
$$

The sets $E \leqq B$ which are of the form $\prod_{\sigma \in S} A_{\sigma}$, with each $A_{\sigma}$ a disjoint pair of elements of $B$, are called $P_{\alpha}$ subsets of $B$.

Proposition 6.2. Let $B$ be an $\alpha$-distributive B.A. and suppose $I$ is

3 Note that since $X(B)$ is compact, every closed set which is an $\alpha$-intersection of open sets is also an $\alpha$-intersection of open-and-closed sets.

${ }_{4}$ This theorem is a special case of known results. (See [1] and the following abstracts from B.A.M.S. vol. 61 (1955): Smith 210, Chang 579, Scott 675 and Tarski 677.) We include the proof for the sake of completeness. The argument is the same as the topological proof of Loomis' theorem, given, for instance in Halmos' Measure Theory, p. 171. 
an $\alpha$-ideal of $B$. Then $B / I$ is $\alpha$-distributive if and only if every $P_{\alpha}$ subset of $B$ which is contained in $I$ has a l.u.b. in $I$.

Proof. Suppose $B / I$ is $\alpha$-distributive. Let $E=\prod_{\sigma \in S} A_{\sigma}$ be a $P_{\alpha}$ set with $E \cong I$. Then

$$
E=\left\{e_{\varphi} \mid \varphi \in F=T^{S}\right\} \cup\{0\} .
$$

where $e_{\varphi}=\bigwedge_{\sigma \in S} a_{\sigma \varphi(\sigma)}, T=[1,2]$. Let $a \rightarrow \bar{a}$ be the natural homomorphism of $B$ onto $B / I$. By the $\alpha$-distributivity of $B / I$,

$$
\bigwedge_{\sigma \in S}\left(\bar{a}_{\sigma 1 \bigvee} \bar{a}_{\sigma 2}\right)=\bigvee_{\varphi \in F} \bar{e}_{\varphi}=\overline{0}
$$

and hence

$$
\bigvee_{\varphi \in F} e_{\varphi} \leqq \bigwedge_{\sigma \in S}\left(a_{\sigma 1 \vee} a_{\sigma 2}\right) \in I
$$

Conversely, suppose $B / I$ is not $\alpha$-distributive. Then by 3.4 , there exists $\bar{b} \neq \overline{0}$ in $B / I$ and

$$
\left\{\overline{C_{\sigma}} \leqq B / I \mid \sigma \in S, \overline{\bar{S}} \leqq \alpha\right\}
$$

such that

$$
\overline{C_{\sigma}}=\left[\bar{c}_{\sigma 1}, \bar{c}_{\sigma_{2}}\right]
$$

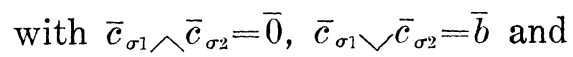

$$
\prod_{\sigma \in S} \bar{C}_{\sigma}=\{0\},
$$

Choose an element $b \in B$ whose image in $B / I$ is $\bar{b}$. Next, pick counterimages

$$
\left[c_{\sigma 1}, c_{\sigma 2}\right] \subseteq B
$$

of the pairs $\bar{C}_{\sigma}$ in such a way that $c_{\sigma 1} \wedge c_{\sigma 2}=0$ and $c_{\sigma 1} \vee c_{\sigma 2}=b$. Then $\prod_{\sigma \in S} C_{\sigma}$ is a $P_{\alpha}$ subset of $B$ which is contained in $I$ and whose least upper bound is $b$ (since $B$ is $\alpha$-distributive), which is not in $I$.

Proposition 6.3. Let $B$ an $\alpha$-B.A. Then a subset $E$ of $B$ is a $P_{a}$ subset if and, assuming $B$ is $\alpha$-distributive, only if

(a) $0 \in E$,

(b) the elements of $E$ are disjoint,

(c) 1.u.b. $E$ exists in $B$,

(d) there exists $E_{\sigma} \leqq E$ defined for each $\sigma$ in an index set $S$ with $\overline{\bar{S}} \leqq \alpha$, such that l.u.b. $E_{\sigma}$ exists for all $\sigma$ and the sets $E_{\sigma}$ distinguish the nonzero elements of $E$, that is, if $e \neq \tilde{e}$ are nonzero elements of $E$, 
then there is an $E_{\sigma}$ which contains $e$ or $\tilde{c}$, but not both.

Proof. The necessity of (a)-(c) is clear from 6.1. The subsets $E_{\sigma}$ of (d) are obtained by letting $E_{\sigma}=\left[e \in E \mid e \leqq a_{\sigma 1}\right]$. Evidently, l.u.b. $E_{\sigma}=a_{\sigma 1 \wedge}$ (l.u.b. $\left.E\right)$.

To show that (a)-(d) are sufficient, let $a=$ l.u.b. $E, a_{\sigma 1}=$ l.u.b. $E_{\sigma}$ and $a_{\sigma_{3}}=$ l.u.b. $\left(E-E_{\sigma}\right)$. By (b), $a_{\sigma_{2}}=a_{\wedge}\left(a_{\sigma_{1}}\right)^{\prime}$ and for $e \in E$, either $e \leqq a_{\sigma 1}$ and $e_{\wedge} a_{\sigma 2}=0$, or vice versa. We prove that $E=\prod_{\sigma \in S} A_{\sigma}$.

Suppose $\varphi \in F$ and $e \in E$ satisfy

$$
e_{\wedge} \bigwedge_{\sigma \in S} a_{\sigma \varphi(\sigma)} \neq 0 \text {. }
$$

Then $e_{\wedge} a_{\sigma \varphi(\sigma)} \neq 0$ for all $\sigma \in S$, so $e \leqq a_{\sigma \varphi(\sigma)}$. Consequently,

$$
e=\bigwedge_{\sigma \in S} a_{\sigma \varphi(\sigma)}
$$

(by (b) and (d)). Thus, $\prod_{\sigma \in, S} A_{\sigma} \leqq E$. On the other hand, for $e \neq 0$ in $E$, define $\varphi \in F$ by $\varphi(\sigma)=1$ if $e \equiv E_{\sigma}, \varphi(\sigma)=2$ if $e \neq E-E_{\sigma}$. Then $0 \neq e=$ $e_{\wedge} \bigwedge_{\sigma \in S} a_{\sigma \varphi(\sigma)}$, and therefore $e=\bigwedge_{\sigma \in S} a_{\sigma \varphi(\sigma)}$. Hence, $E \leqq \prod_{\sigma \in S} A_{\sigma}$.

Corollary 6.4. Let $B$ be a $2^{\alpha}$-B.A. Then $E \subseteq B$ is a $P_{\alpha}$ subset if and only if. $0 \in E$, the elements of $E$ are disjoint and $\overline{\bar{E}} \leq 2^{\alpha}$.

Proof. The necessity is clear. To prove the sufficiency, observe that since $\overline{\bar{E}} \leq 2^{\alpha}$, it is possible to find a one-to-one map $\lambda$ of $E$ into the set of all two-valued functions on a set $S$ of cardinality $\leq \alpha$. For each $\sigma \in S$, let

$$
E_{\sigma}=\{e \in E \mid[\lambda(e)](\sigma)=1\} .
$$

It is clear that the system $\left\{E_{\sigma} \mid \sigma \subseteq S\right\}$ satisfies condition (d) of 6.3.

Corollary 6.5. Let $B$ be a $2^{\alpha}$-B.A. which is $\alpha$-distributive. Let $I$ be an $\alpha$-ideal of $B$. Then $B / I$ is $\alpha$-distributive if and only if $I$ is $a 2^{\alpha}$ ideal.

Proof. ${ }^{5}$ If $C \leqq I$ satisfies $\overline{\bar{C}} \leqq 2^{\alpha}$, then using Zorn's lemma, it is possible to find a set $E$ of disjoint elements such that $\overline{\bar{E}} \leq \overline{\bar{C}}$, l.u.b. $E$ =l.u.b. $C$ and every $e \in E$ is contained in some $c \in C$ (so that $E \subseteq I$ ). By 6.4, $E \cup[0]$ is a $P_{\alpha}$ subset of $B$. By 6.2, l.u.b. $(E \cup[0]) \in I$. Thus, l.u.b. $C \in I$.

${ }^{5}$ See footnote 2 . As noted in Smith and Tarski [5], the assumption that $B$ is $\alpha$ distributive in 6.6 is unnecessary. This condition was used only to prove the sufficiency in 6.2 . 
CoRollary 6.6. Let $B$ be a $2^{\alpha}$-complete, $\alpha$-distributivite B.A. Suppose $\alpha$ is weakly accessible from the infinite cardinal $\beta$. Let $I$ be $a \beta$ $i d e a l$ of $B$ such that $B / I$ is $\alpha$-distributive. Then $I$ is a $2^{\alpha}$-ideal.

Proof. First, observe that if $\xi$ is a singular cardinal and $I$ is an $\eta$-ideal for all $\eta<\xi$, then $I$ is a $\xi$-ideal. Using this fact, 6.6 follows from 6.5 by transfinite induction on $\alpha$.

It should be remarked that the methods and results of this section are related to the circle of ideas developed by Ulam and Tarski in [9] and [8]. For example, it follows directly from 6.6 that if $B$ is a $2^{\alpha}$ field, where $\alpha$ is weakly accessible from $\beta$, then any prime $\beta$-ideal is also a $2^{\alpha}$-ideal (see [8], Theorem 3.19).

7. The lattice of continuous functions on $X(B)$. Stone has proved (see [7], p. 186) that a Boolean algebra $B$ is $\alpha$-complete if and only if the lattice of real valued, continuous functions on its Boolean space is conditionally $\alpha$-complete. This result immediately suggests the

Theorem 7.1. Let $B$ be a Boolean algebra. Then $B$ is $\alpha$-distributive if and only if the lattice $C(X(B))$ of real valued, continuous functions on the Boolean space of $B$ is $\alpha$-distributive ${ }^{6}$.

Proof. Assume first that $C(X(B))$ is conditionally $\alpha$-complete. Then the set of all characteristic functions of open-and-closed subsets of $X(B)$ form an $\alpha$-sublattice of $C(X(B))$ which is clearly lattice isomorphic to $B$ (see the proof of Theorem 12 of [7]). Consequently, if $C(X(B))$ is $\alpha$ distributive, so is $B$.

Conversely, suppose $B$ is $\alpha$-distributive (and $\alpha$-complete). Then by Stone's result, cited above, $C(X(B))$ is conditionally $\alpha$-complete and we have only to verify the relation (1) of 1.1 .

First consider the special case where each function $a_{\sigma r}$ takes only finitely many real values. Let $A_{\sigma \tau}=\left\{b_{\sigma \tau n} \mid n=1,2, \cdots\right\}$ be a finite set of disjoint elements of $B$ such that $\bigvee_{n} b_{\sigma \tau n}=u$ and $a_{\sigma r}$ is constant on each set $X\left(b_{\sigma r n}\right)$. By 3.2 , there is a covering $A$ of $B$ such that $A$ refines every $A_{\sigma \tau}$. If $b \in A$, then every $a_{\sigma \tau}$ is constant on $X(b)$. Since $a \rightarrow$ $\left(a|X(b), a| X\left(b^{\prime}\right)\right)$ is a direct decomposition of $C(X(B))$, the restriction homomorphism $\pi_{b}: a \rightarrow a \mid X(b)$ preserves arbitrary joins and meets. Moreover, $\pi_{b}$ sends all $a_{\sigma \tau}$ into the conditionally complete sublattice of constant functions on $X(b)$. This sublattice, being isomorphic to the chain of real numbers, is evidently $\alpha$-distributive. Hence,

${ }^{6}$ That is, $C(X(B))$ is a conditionally $\alpha$-complete lattice which satisfies the identity (1) of Definition 1.1 when the elements $a_{\sigma \tau}$ are functions which have a common upper bound and a common lower bound. 


$$
\bigwedge_{\sigma \in S} \bigvee_{\tau \in T} \pi_{l}\left(a_{\sigma \tau}\right)=\bigvee_{\varphi \in F} \bigwedge_{\sigma \in S} \pi_{b}\left(a_{\sigma \varphi(\sigma)}\right) .
$$

Using this remark, we show that $\wedge_{\sigma} \bigvee_{\tau} a_{\sigma_{\tau}}$ is the least upper bound in $C(X(B))$ of the set $\left\{\bigwedge_{\sigma \in s} a_{\sigma \varphi(\sigma)} \mid \varphi \in T^{s}\right\}$.

Suppose $f \geqq \bigwedge_{\sigma} a_{\sigma \mathscr{\varphi}(\sigma)}$ for all $\varphi$. The if $b \in A, \pi_{b}(f) \geqq \bigwedge_{\sigma} \pi_{b}\left(a_{\sigma \varphi(\sigma)}\right)$ for all $\varphi$, so

$$
\pi_{b}(f) \geqq \bigvee_{\varphi} \bigwedge_{\sigma} \pi_{b}\left(a_{\sigma \varphi(\sigma)}\right)=\bigwedge_{\sigma} \bigvee_{\tau} \pi_{b}\left(a_{\sigma \tau}\right)=\pi_{b}\left(\bigwedge_{\sigma} \bigvee_{\tau} a_{\sigma_{\tau}}\right)
$$

Thus $f(P) \geqq\left(\bigwedge_{\sigma} \vee_{\tau} a_{\sigma \tau}\right)(P)$ pointwise on the dense set $\bigcup_{b \in A} X(b)$ and therefore, by continuity, $f \geqq \wedge_{\sigma} \vee_{\tau} a_{\sigma \tau}$. By definition of the least upper bound, $\wedge_{\sigma} \bigvee_{\tau} a_{\sigma \tau}=\bigvee_{\varphi} \bigwedge_{\sigma} a_{\sigma \varphi(\sigma)}$.

Now consider the general case of arbitrary functions $a_{\sigma_{\tau}}$. Since $X(B)$ is compact and totally disconnected, the Stone-Weierstrass theorem guarantees the existence (for each $\sigma \in S, \tau \in T$ and integer $n$ ) of functions $f_{\sigma \tau}$, taking only finitely many real values, such that $\left|f_{\sigma \tau}-a_{\sigma \tau}\right| \leqq 1 / n$. Suppose $f \in C(X(B))$ satisfies $f \geqq \wedge_{\sigma} a_{\sigma \omega(\sigma)}$ for all $\varphi \in T^{S}$. Then

$$
f \geqq \wedge_{\sigma}\left(f_{\sigma \varphi(\sigma)}-1 / n\right)
$$

for all $\varphi$. Hence, by the result of the special case,

$$
\begin{aligned}
f & \geq \bigvee_{\varphi} \bigwedge_{\sigma}\left(f_{\sigma \varphi(\sigma)}-1 / n\right)=\left(\bigvee_{\varphi} \bigwedge_{\sigma} f_{\sigma \varphi(\sigma)}\right)-1 / n=\left(\wedge_{\sigma} \bigvee_{\tau} f_{\sigma \tau}\right)-1 / n \\
& \geq\left(\bigwedge_{\sigma} \bigvee_{\tau}\left(a_{\sigma_{\tau}}-1 / n\right)\right)-1 / n=\left(\bigwedge_{\sigma} \bigvee_{\tau} a_{\sigma_{\tau}}\right)-2 / n .
\end{aligned}
$$

Since $n$ can be arbitrarily large, $f \geqq \wedge_{\sigma} \vee_{\tau} a_{\sigma_{\tau}}$. Thus, $\wedge_{\sigma} \vee_{\tau} a_{\sigma_{\tau}}=$ $\bigvee_{\varphi} \bigwedge_{\sigma} a_{\sigma \varphi(\sigma)}$.

8. The continuous functions on $X(B)$. In this section we consider the individual continuous functions on the Boolean space of an $\boldsymbol{S}^{-}$ distributive B.A.

Lemma 8.1. Let $B$ be an sto-distributive B.A. Let $X(B)$ be the Boolean space of $B$. Let $Y$ be a separable metric space. Then any continuous mapping $f$ of $X(B)$ into $Y$ is locally constant on a dense subset of $X(B)$, that is, the set of points $P$ of $X(B)$ such that $f$ is constant on some neighborhood of $P$ is dense in $X(B)$.

Proof. Let $\left\{N_{1}, N_{2}, \cdots, N_{n}, \cdots\right\}$ be a countable neighborhood basis of $Y$. Set $M_{n}=f^{-1}\left(N_{n}\right)$. Since $Y$ is a metric space, $N_{n}$ is an open $F_{\sigma}$ (that is, a countable union of closed sets). By the continuity of $f$, so is $M_{n}$. But $X(B)$ is the Boolean space of an $\psi_{0}$-B.A., so the closure of any open $F_{\sigma}$ in $X(B)$ is open (see [5], Theorems 17 and 18). Hence, elements $b_{n} \in B$ exist so that $M_{n}^{-}=X\left(b_{n}\right)$.

Let $A_{n}=\left[b_{n}, b_{n}^{\prime}\right]$. Then there is a covering $A$ of $B$ which refines all $A_{n}$. By 5.2, $\cup_{a \in A} X(a)$ is dense in $X(B)$. It will be sufficient to prove 
that $f$ is constant on $X(a)$ for each $a \in A$.

Suppose $f(P) \neq f(Q)$. Then there exists $N_{n}$ such that $f(P) \in N_{n}$, $f(Q) \notin N_{n}^{-}$. Thus $P \in M_{n} \leqq X\left(b_{n}\right)$, but $Q \notin M_{n}^{-}$, since $f\left(M_{n}^{-}\right) \subseteq N_{n}^{-}$. Hence, $Q \in X\left(b_{n}^{\prime}\right)$. Consequently, $P$ and $Q$ cannot lie in the same set $X(a)$ with $a \in A$. In other words, $f$ is constant on each $X(a)$.

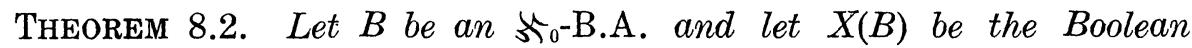
space of. $B$. Then a necessary and sufficient condition that $B$ be s.distributive is that every real valued, continuous function on $X(B)$ be locally constant on a dense subset of $X(B)$.

Proof. Necessity is a special case of 8.1. Suppose then that every real valued continuous function is locally constant on a dense set. Let $A_{n}=\left[a_{n}, a_{n}^{\prime}\right]$ be a countable set of binary partitions of $B$. Let $\psi_{n} \in$ $C(X(B))$ be defined by $\psi_{n}(P)=0$ if $P \in X\left(a_{n}\right), \psi_{n}(P)=2$ if $P \in X\left(a_{n}^{\prime}\right)$. Set $f(P)=\sum_{n=1}^{\infty} \psi_{n}(P) / 3^{n}$. Then $f$ is continuous on $X(B)$. Note that $f(P)=$ $f(Q)$ if and only if $\psi_{n}(P)=\psi_{n}(Q)$ for all $n$ (because the points of the Cantor set have unique representations in the form $\sum_{n=1}^{\infty} \delta_{n} / 3^{n}$ with $\delta_{n}=$ $0,2)$. By assumption, $f$ is locally constant on a dense set. Thus, there is a subset $A$ of $B$ such that $\bigcup_{a \in A} X(a)$ is dense in $X(B)$ and $f$ is constant on each $X(a)$ with $a \in A$. This implies $A$ is a covering of $B$ and every $\psi_{n}$ is constant on each $X(a)$, so that $A$ refines every $A_{n}$. By 3.2, $B$ is $\widehat{H}_{0}$-distributive.

\section{Unsolved problems,}

(9.1) What properties of the Boolean space of $B$ characterize $\alpha$ distributivity? One can deduce from 3.4 the following result, which, seemingly, is only slightly weaker than the converse of $5.3:$ if $B$ is an $\alpha$-B.A. which is not $\alpha$-distributive, then there is a nonempty open subset of $X(B)$ which is contained in a $2^{\alpha}$-union of $\alpha$-nowhere dense sets.

(9.2) Is the completion by Dedekind cuts of an $\alpha$-field (or more generally, and $\alpha$-distributive B.A.) itself $\alpha$-distributive?

(9.3) Is every $2^{\alpha}$-complete, $\alpha$-distributive B.A. the $2^{\alpha}$-homomorph of a $2^{\alpha}$-field? By 6.5 , it would be enough to prove that every $\alpha$-distributive, $2^{\alpha}$-B.A. is the $\alpha$-homomorph of a $2^{\alpha}$-field.

\section{References}

1. P. Erdös and A. Tarski, On families of mutually exclusive sets, Ann. of Math, 44 (1943), 315-329. 
2. A. Horn and Tarski, Measures in Boolean algebrus, Trans. Amer. Math. Soc. 64 (1948), 467-497.

3. L. H. Loomis, On the representation of o-complete Boolean algebras, Bull. Amer. Math. Soc. 53 (1947), 757-760.

4. R. Sikorski, On the representation of Boolean algebras as fields of sets, Fund. Math. 35 (1948), 247-258.

5. E. C. Smith and A. Tarski, Higher degrees of distributivity and completeness in Boolean algebras, to appear in Trans. Amer. Math. Soc.

6. M. H. S sone, Applications of the theory of Boolean rings to general topology, Trans. Amer. Math. Soc. 41 (1937), 375-481.

7. - - Boundedness properties in function-lattices, Canad. J. Math. 1 (1949), 176-186.

8. A. Tarki, Über additive und multiplikative Mengenkörper und Mengenfunktionen, Soc. Sci. Lett. Varsovie C.R. Cl. III, 30 (1937), 151-181.

9. S. Ulam, Zur Masstheorie in der allgemeinen Mengenlehre, Fund. Math. 16 (1930), 140-150.

\section{UNIVERSITY OF WASHINGTON}




\section{PACIFIC JOURNAL OF MATHEMATICS}

EDITORS

H. L. ROYDEN

Stanford University

Stanford, California

R. A. Beaumont

University of Washington

Seattle 5 , Washington
A. R. Whiteman

University of Southern California

Los Angeles 7, California

E. G. Straus

University of California

Los Angeles 24, California

\section{ASSOCIATE EDITORS}
E. F. BECKENBACH
C. E. BURGESS
M. HALL
E. HEWITT
A. HORN
V. GANAPATHY IYER
R. D. JAMES
M. S. KNEBELMAN

L. NACHBIN

I. NIVEN

G. SZEKERES

T. G. OSTROM

M. M. SCHIFFER
F. WOLF

K. YOSIDA

\section{SUPPORTING INSTITUTIONS}

UNIVERSITY OF BRITISH COLUMBIA

CALIFORNIA INSTITUTE OF TECHNOLOGY

UNIVERSITY OF CALIFORNIA

MONTANA STATE UNIVERSITY

UNIVERSITY OF NEVADA

OREGON STATE COLLEGE

UNIVERSITY OF OREGON

UNIVERSITY OF SOUTHERN CALIFORNIA

\author{
STANFORD UNIVERSITY \\ UNIVERSITY OF UTAH \\ WASHINGTON STATE COLLEGE \\ UNIVERSITY OF WASHINGTON \\ AMERICAN MATHEMATICAL SOCIETY \\ CALIFORNIA RESEARCH CORPORATION \\ HUGHES AIRCRAFT COMPANY \\ THE RAMO-WOOLDRIDGE CORPORATION
}




\section{Pacific Journal of Mathematics}

\section{Vol. 7, No. $1 \quad$ January, 1957}

Richard Davis Anderson, Zero-dimensional compact groups of

homeomorphisms ................................... 797

Hans-Joachim Bremermann, Holomorphic functionals and complex

convexity in Banach spaces........................... 811

Hugh D. Brunk, G. M. Ewing and W. R. Utz, Minimizing integrals in

certain classes of monotone functions ................. 833

Philip David, Uniqueness theory for asymptotic expansions in general

regions ...................................... 849

Paul Erdős and Harold Nathaniel Shapiro, On the least primitive root of a

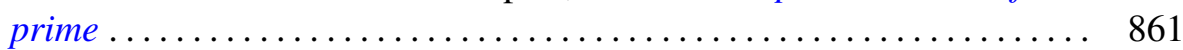

Watson Bryan Fulks, Regular regions for the heat equation ........... 867

William Robert Gaffey, A real inversion formula for a class of bilateral

Laplace transforms ................................ 879

Ronald Kay Getoor, On characteristic functions of Banach space valued random variables ................................. 885

Louis Guttman, Some inequalities between latent roots and minimax (maximin) elements of real matrices ...................... 897

Frank Harary, The number of dissimilar supergraphs of a linear graph .... 903

Edwin Hewitt and Herbert S. Zuckerman, Structure theory for a class of convolution algebras .................................. 913

Amnon Jakimovski, Some Tauberian theorems . . . . . . . . . . . . . . . . . 943

C. T. Rajagopal, Simplified proofs of "Some Tauberian theorems" of Jakimovski................................

Paul Joseph Kelly, A congruence theorem for trees ................. 961

Robert Forbes McNaughton, Jr., On the measure of normal formulas...... 969

Richard Scott Pierce, Distributivity in Boolean algebras .............. 983

Calvin R. Putnam, Continuous spectra and unitary equivalence ......... 993

Marvin Rosenblum, Perturbation of the continuous spectrum and unitary

equivalence................................... 997

V. N. Singh, Certain generalized hypergeometric identities of the

Rogers-Ramanujan type.......................

Peter Swerling, Families of transformations in the function spaces $H^{p} \ldots \ldots 1015$ 